\title{
CORRESPONDENCE
}

\section{Severe Measles Pneumonitis in Adults}

SiR - We read with interest the report by Forni et al. [1] describing six cases of severe measles pneumonitis in adults. They describe myositis as an uncommon clinical feature of the disease and provide circumstantial evidence for the efficacy of intravenous ribavarin therapy in this clinical setting. We recently cared for two adult patients with severe measles; one of these patients evidenced marked elevation of creatine phosphokinase (CPK) activity, and the second, whose infection was acquired nosocomially, developed pneumonitis requiring mechanical ventilation. A third case, which was also nosocomial, was mild.

Patient 1. A 24-year-old Swiss male presented with fever, diarrhea, dyspnea, and a cough upon return from a vacation in the Philippines. On physical examination his temperature was $39.2^{\circ} \mathrm{C}$ and his respirations were $24 / \mathrm{min}$; a macular, disseminated rash and conjunctival injection were noted. There were no muscular signs or symptoms. Laboratory values are shown in table 1. Findings on chest roentgenogram were normal. After some delay, a diagnosis of measles was made. The patient's respiratory symptoms promptly resolved with administration of oxygen by nasal cannula for 24 hours, and he became afebrile within 48 hours. He was discharged on day 5 . A serological test was strongly positive for IgM antibodies to measles virus.

Patient 2. A 34-year-old nurse who cared briefly for patient 1 in the emergency department developed a fever (temperature $>39^{\circ} \mathrm{C}$ ) 9 days later; the next day she noted a rash on her face. Twelve days after exposure she presented with a temperature of $38.3^{\circ} \mathrm{C}$, a dry cough, conjunctival and pharyngeal injection, and a generalized macular rash. Two days later ( 5 days after her symptoms first appeared) she was hospitalized because of persistent fever (temperature to $40^{\circ} \mathrm{C}$ ) and dyspnea. Chest roentgenography showed generalized bilateral alveolar infiltrates. On day 10 of the illness, the patient was intubated because of severe respiratory insufficiency. Amoxicillin/clavulanic acid was administered for presumed bacterial superinfection. Mechanical ventilation was continued for 3 days; the patient recovered uneventfully and was discharged home after a hospital stay of 16 days. Seroconversion for measles virus-specific antibodies occurred.

Patient 3. The radiology technician who performed chest roentgenography for patient 1 developed mild clinical measles 10 days later. None of the three patients had a history of measles or measles vaccination.

We could not explain the eightfold increase in the CPK level in patient 1 until we read the article by Forni et al. [1], in which the possible explanation of measles-induced myositis was provided. The possibility of an exercise-induced elevation of the CPK level that could have been triggered by the patient's respiratory

Reprints or correspondence: Dr, Jacques Gubler, Medizinische Klinik, Stadtspital Triemli, Birmensdorferstrasse 497, CH - 8063 Zürich, Switzerland.

Clinical Infectious Diseases 1995;21:1060-1

(C) 1995 by The University of Chicago. All rights rescrved.

$1058-4838 / 95 / 2104-0069 \$ 02.00$
Table 1. Laboratory data for two patients with measles pneumonitis.

\begin{tabular}{|c|c|c|}
\hline Parameter & Patient 1 & Patient 2 \\
\hline $\mathrm{PO}_{2}(\text { normal, }>9.0 \mathrm{kPa})^{*}$ & $5.1 \mathrm{kPa}$ & $4.8 \mathrm{kPa}$ \\
\hline $\mathrm{PCO}_{2}$ (normal, $5.0-6.0 \mathrm{kPa}$ ) & $4.6 \mathrm{kPa}$ & $5.8 \mathrm{kPa}$ \\
\hline Hemoglobin level & $15.2 \mathrm{~g} / \mathrm{dL}$ & $14.0 \mathrm{~g} / \mathrm{dL}$ \\
\hline Leukocyte count & $7,100 / \mathrm{mm}^{3}$ & $4,400 / \mathrm{mm}^{3}$ \\
\hline Thrombocyte count & $141,000 / \mathrm{mm}^{3}$ & $96,000 / \mathrm{mm}^{3}$ \\
\hline $\begin{array}{l}\text { Aspartate aminotransferase leve } \\
\text { (normal, }<40 \mathrm{U} / \mathrm{L} \text { ) }\end{array}$ & $124 \mathrm{U} / \mathrm{L}$ & $50 \mathrm{U} / \mathrm{L}$ \\
\hline $\begin{array}{l}\text { Alanine aminotransferase level } \\
\text { (normal, }<40 \mathrm{U} / \mathrm{L} \text { ) }\end{array}$ & $19 \mathrm{U} / \mathrm{L}$ & $26 \mathrm{U} / \mathrm{L}$ \\
\hline $\begin{array}{l}\text { Lactate dehydrogenase level } \\
\text { (normal, }<450 \mathrm{U} / \mathrm{L} \text { ) }\end{array}$ & $1,863 \mathrm{U} / \mathrm{L}$ & $1,563 \mathrm{U} / \mathrm{L}$ \\
\hline $\begin{array}{l}\text { Creatinine phosphokinase level } \\
\text { (normal, } 10-190 \mathrm{U} / \mathrm{L} \text { ) }\end{array}$ & $2,082 \mathrm{U} / \mathrm{L}$ & $142 \mathrm{U} / \mathrm{L}$ \\
\hline
\end{tabular}

* While breathing room air.

effort in response to hypoxemia must be excluded since elevation of the CPK levels persisted longer than did his respiratory distress. This patient's hypoxemia can best be explained by the presence of alveolitis that resolved spontaneously; findings on a chest roentgenogram were unremarkable, and results of blood gas analysis became normal within 36 hours. It is conceivable that myositis has been unnoticed in previous cases because CPK studies were not done routinely or because elevations in levels of aspartate aminotransferase and lactate dehydrogenase were interpreted as representing hepatitis when they in fact reflected myositis [2].

The case of patient 2 demonstrates that severe measles pneumonitis in immunocompetent adults, even when intubation and mechanical ventilation are required, can have a favorable course in the absence of specific therapeutic measures. Despite some evidence for an effect of ribavarin in patients with measles [3, 4], the drug's value in adults has yet to be convincingly demonstrated. Finally, this case, in which severe measles was acquired in the hospital setting, reminds us of the potential hazard of nosocomial measles and the need for strict adherence to recommendations for immunization of hospital employees $[5,6]$.

Jacques Gubler, Regina Lüthy, and Oswald Oelz Department of Medicine, Stadtspital Triemli, Zürich, Switzerland

\section{References}

1. Forni AL, Schluger NW, Roberts RB. Severe measles pneumonitis in adults: evaluation of clinical characteristics and therapy with intravenous ribavarin. Clin Infect Dis 1994; 19:454-62.

2. Gremillion DH, Crawford GE. Measles pneumonia in young adults: an analysis of 106 cases. Am J Med 1981;71:539-42.

3. Shigeta $S$, Mori $S$, Baba M, et al. Antiviral activities of ribavirin, 5-ethynyl$1-\beta$-D-ribofuranosylimidazole-4-carboxamide, and $6^{\prime}$-(R)-6'-C-methylneplanocin A against several ortho- and paramyxoviruses. Antimicrob Agents Chemother 1992;36:435-9. 
4. Uylangco CV, Beroy GJ, Santiago LT, Mercoleza VD, Mendoza SL. $\Lambda$ double-blind, placebo-controlled evaluation of ribavirin in the treatment of acute measles. Clin Ther 1981;3:389 96 .

5. Davis RM, Orenstein WA, Frank JA Jr, et al. Transmission of

\section{Bronchoesophageal Fistula Due to Multidrug-Resistant Tuberculosis in a Patient Infected with Human Immunodeficiency Virus}

SIR-Bronchoesophageal fistulas are classified as congenital or acquired; the majority of those seen in adults are acquired. The causes include malignancy, trauma, and infection. The infectious agents that are responsible for these fistulas include Mycobacterium tuberculosis, Histoplasma capsulatum, Actinomyces species, and Treponema pallidum. Tuberculosis is the most common cause of inflammatory fistulas [1].

Porter et al. noted that $<30$ cases of tuberculous bronchoesophageal fistulae (BEF) have been reported in the literature, and they described three additional patients who were infected with HIV [2]. All three of their patients were from areas where tuberculosis is endemic and had CD4 lymphocyte counts of $20 / \mathrm{mm}^{3}$, and all three were infected with organisms that were susceptible to all antituberculous drugs; two of the three patients had documented medical response with closure of the fistula (one was lost to followup). We also treated an HIV-infected patient who had a BEF due to $M$. tuberculosis; however, this case had unique features.

A 48-year-old homosexual man from Argentina who was infected with HIV presented to the hospital with a 1-week history of cough, dyspnea, and fever (temperature, $38.4^{\circ} \mathrm{C}$ ). Physical examination revealed clear lungs and a nontender supraclavicular lymph node. Chest radiographs demonstrated bilateral interstitial and alveolar infiltrates. The patient received pentamidine for presumed Pneumocystis carinii pneumonia. His condition initially improved, but then the infiltrates became more extensive and he developed dysphagia.

Bronchoscopy, which was performed on hospital day 20 , revealed a $7-\mathrm{mm}$ opening in the right main-stem bronchus below the main carina, and polypoid white and black tissue was visible. Histopathologic examination revealed squamous epithelium-lined mucosa that was acutely and chronically inflamed; there were no granulomas, but numerous acid-fast bacilli (AFB) were observed. Transbronchial biopsy showed mild interstitial fibrosis, and Grocott-Gomori methenamine-silver nitrate stains were negative for P. carinii but positive for AFB. A barium swallow showed a BEF below the level of the carina, with extravasation of barium into the right bronchial tree. A CT scan of the chest revealed bilateral infiltrates, mediastinal lymphadenopathy, and an air pocket corresponding to the level of the fistula. A biopsy of the supraclavicular lymph node was also performed, and a stain for AFB was positive.

The attending physician was then notified that the patient had had multidrug-resistant tuberculosis (MDRTB) and that the $M$.

Reprints or correspondence: Dr. Deborah S. Asnis, Flushing Hospital Medical Center, 4500 Parsons Boulevard, Flushing, New York 11355.

Clinical Infectious Diseases 1995;21:1061-2

(C) 1995 by The University of Chicago. All rights reserved.

$1058-4838 / 95 / 2104-0070 \$ 02.00$ measles in medical settings, 1980 through 1984. J $\Lambda$ MA 1986; 255:1295-8.

6. Immunization Practices Advisory Committee. Measles prevention, MMWR Morb Mortal Wkly Rep 1989:38(SS-9):1 17.

tuberculosis isolate had been resistant to isoniazid, rifampin, ethambutol, and pyrazinamide); he was treated with intravenous isoniazid $(300 \mathrm{mg}$ once a day), oral ethionamide $(250 \mathrm{mg}$ every 8 hours), intravenous amikacin ( $350 \mathrm{mg}$ every 12 hours), oral rifabutin (300 $\mathrm{mg}$ every day), and oral cycloserine ( $250 \mathrm{mg}$ every 8 hours). He received total parenteral nutrition and was permitted to take only medications orally. Multidrug-resistant $M$. tuberculosis was cultured from sputum, bronchoscopic washings, the supraclavicular lymph node, and gastric washings. The patient continued to receive rifabutin, ethionamide, cycloserine, and amikacin, but therapy with isoniazid was stopped. After 4 weeks, a repeated barium swallow showed complete closure of the fistula, and the patient resumed oral feedings without any intolerance. He was discharged from the hospital 4 months after admission.

In the past few years, there have been numerous outbreaks of MDRTB. Most cases of MDRTB have been in patients infected with HIV [3]. MDRTB can occur as a result of infection by organisms that arc already resistant to antituberculous drugs or as a result of the development, during treatment, of drug resistance in a strain that was not present initially. It was previously believed that infection with $M$. tuberculosis would protect against reinfection with the same organism because of acquired immunity. One study [4] described five HIV-infected patients who were documented by restriction fragment length polymorphism to be infected with initially susceptible isolates but who developed MDRTB during treatment. It was concluded that they must have been exogenously reinfected with a new strain of $M$. tuberculosis [4].

The mortality among patients with MDRTB has ranged from $72 \%$ to $89 \%$, and the median interval between the diagnosis of MDRTB and death has been short, ranging from 4 to 6 weeks [3]. The median hospital stay for patients with MDRTB is $>7$ months [5]. Not only did our patient's condition improve, but he survived after developing a devastating complication due to MDRTB and his fistula closed as well. Follow-up cultures for $M$. tuberculosis were negative. He died of progressive Kaposi's sarcoma and dementia 2 months after discharge. Porter et al. stress that a diagnosis of tuberculosis should be considered for HIV-infected individuals with associated intrathoracic lymphadenopathy, fever, and dysphagia and that antituberculous chemotherapy should be administered pending culture results [2]. The possibility of MDRTB-induced BEF should be kept in mind in areas where $M$. tuberculosis is endemic. With appropriate antituberculous therapy and nutritional support, a patient with an MDRTB-induced BEF should respond, resulting in clearance of the infection as well as healing of the fistula.

Deborah S. Asnis, Herbert P. Saltzman, and Jose A. Giron Department of Internal Medicine, Flushing Hospital Medical Center, Flushing, New York

\section{References}

1. Spalding AR, Burney DP, Richie RE. Acquired benign bronchoesophageal fistulas in the adult. Ann Thorac Surg 1979;28:378 83. 